\title{
Critical analysis of the definition of severe dengue, based on the Ecuador outbreak in 2012
}

\author{
Natalia Romero Sandoval ${ }^{1,2,3}$, Pablo Lozano ${ }^{3,4}$, Jacqueline Cevallos ${ }^{3,4}$, Virginia Ruiz ${ }^{1,3}$, \\ Miguel Martín $3,5,6$

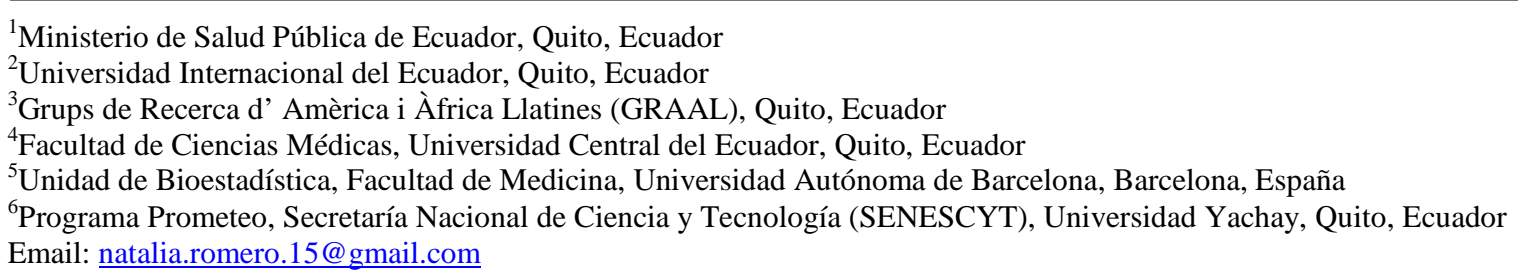

Received 1 January 2014; revised 1 February 2014; accepted 8 February 2014

Copyright (C) 2014 Natalia Romero Sandoval et al. This is an open access article distributed under the Creative Commons Attribution License, which permits unrestricted use, distribution, and reproduction in any medium, provided the original work is properly cited. In accordance of the Creative Commons Attribution License all Copyrights (C) 2014 are reserved for SCIRP and the owner of the intellectual property Natalia Romero Sandoval et al. All Copyright (c) 2014 are guarded by law and by SCIRP as a guardian.

\begin{abstract}
Of the 238 cases reported to the Ecuador Health Authorities in 2012 as severe dengue, only $53.3 \%$ met diagnostic criteria. The definition of severe dengue should be based on more precise criteria. Information about compliance with criteria should be fed back to local levels.
\end{abstract}

\section{KEYWORDS}

\section{Severe Dengue; Dengue Hemorrhagic Fever; Fatality Rate}

\section{INTRODUCTION}

According to the Pan-American Health Organization, dengue in America has become steadily more common over the last 25 years and requires a review of health policies [1]. The classification of forms of dengue proposed by the World Health Organization (WHO) in 1997 consists of three categories: undifferentiated fever, dengue fever (classic dengue), and dengue hemorrhagic fever. The latter category includes dengue shock syndrome [2]. This categorization was revised in 2009. The terms dengue with/without warning signs and severe dengue were introduced. It was recommended that the earlier terminology be abandoned [3].

Comparative studies of the impact for health systems of the two classifications suggest that the revised classification, although it discriminates unnecessary interventions, associates the categories of patients with specific instructions for treatment and makes the key therapeutic messages easily understood by health personnel [4]. It doubles their workload and makes laboratory confirmation necessary [5]. And, there is another aspect: the assessment of fatality rate, an important indicator in any outbreak. There has been debate over the denominator to use in constructing this indicator for dengue. Sometimes the denominator includes all cases classified clinically and epidemiologically as dengue. To ameliorate this problem, it would be advisable to use the category of severe dengue, according to the latest classification, as individuals thus classified are the ones most at risk of dying. Defining this category clearly has repercussions for the assessment of outbreak severity since it has a direct influence on the indicator of fatality.

In Ecuador, $70 \%$ of the territory has conditions of temperature, humidity and presence of the vector conducive to development of the disease. In 2012, dengue increased by $216 \%$; severe dengue increased by $315 \%$ and deaths increased by 144\%, compared to 2011 [6]. This situation prompted us to critically analyze the definition of severe dengue.

\section{METHOD}

Between September and December 2012, we analyzed data from 238 patients hospitalized with severe dengue between January and August of the same year. These patients were recorded in 257 clinical records in the hospitals of the affected areas. These data constituted $87.4 \%$ of the cases declared officially. A database was set up with 8641 entries and approximately 100 variables, based on the medical records (origin of event, interven- 
tion in primary care, clinical review, laboratory tests, treatment). All events of these patients were being ordered by date and time. The project was approved by the Ministry of Health of Ecuador. The protocol was submitted and approved by the General Research Committee of Ministry of Health. Confidentiality was ensured by assigning a code to each patient.

The variables used to define probable dengue were fever, headache, pain behind the eyes, nausea, vomiting, generalized pain, skin rash and leucopenia. The warning signs (revised classification) considered were abdominal pain or tenderness, mucosal bleed, liver enlargement, pleural effusion, ascites and lethargy. Patients were considered to have severe dengue if they had plasma leakage (raised hematocrit, decreased platelet count, clinical fluid accumulation) and/or laboratory confirmation (by ELISA IgM or IgG); and in these the presence of impaired consciousness, encephalitis, respiratory distress and/or severe bleeding (recoded based on need for transfusion of blood and severe liver damage-AST or ALT > 1000 IU). In order to identify cases of dengue hemorrhagic fever, in addition to the variables already mentioned, "hemorrhagic manifestations" were included (ecchymosis, purpura, mucosal bleed) and thrombocytopenia. The variable shock was used both in the construction of dengue shock syndrome and severe dengue. Two categories were used for discharge status (alive/dead). The results are expressed as percentages, and the ratio of fatalities was calculated.

\section{RESULTS}

Of the 238 patients studied, 47.9\% were males, 29.0\% aged between 0 and 5 years. 8\% (19/238) appeared in more than one clinical record. The criteria for probable dengue fever were met by $89.5 \%$ of the patients; of these,
$62.9 \%(134 / 213)$ had laboratory confirmation. Criteria for dengue hemorrhagic fever (old classification) were met by $53.8 \%(128 / 238)$ patients and $50.8 \%$ of these met the criteria for dengue shock syndrome.

According to the revised classification, 90.7\% (216/ 238) of the patients met criteria for probable dengue. Plasma leakage and/or laboratory confirmation was documented in 89.1\% (212/238) cases, and 53.3\% (113/212) met criteria for severe dengue (Table 1 ).

Case fatality among the 238 patients declared as having severe dengue was $6.7 \%$. When strict criteria for severe dengue were taken into account, the number of patients dropped to nearly half, and hence the 13 deceased patients who met this definition resulted in a case fatality of $11.5 \%$. Among the patients with dengue hemorrhagic fever case fatality was $7.0 \%$ (Table 2 ).

\section{DISCUSSION}

All patients in this analysis were declared to have severe dengue by the hospitals providing the cases. But we found that only $53.3 \%$ of them met criteria for severe dengue and that their fatality was $75 \%$ higher $(11.5 \%$ vs. $6.7 \%$ ) than the figure which would have been declared based on these cases.

The important difference between reports and a later analysis rigorously applying criteria showed, in a study in Thailand, that of 323 patients hospitalized for dengue, $70.6 \%$ met criteria for dengue hemorrhagic fever and that $27 \%$ met criteria for severe dengue [7].

These variations obviously led to very different fatality rates, since considering that the number of deaths was the same for the different classifications. The fatality ratios ranged from 2.5 to 3.6 .

Table 1. Classification of patients reported as having severe dengue, in terms of the 1997 classification scheme, and the revised scheme.

\begin{tabular}{|c|c|c|c|c|c|}
\hline \multirow{3}{*}{ Definition of case } & \multicolumn{4}{|c|}{ Compliancewithcriteria } & \multirow[b]{3}{*}{ Total } \\
\hline & \multicolumn{2}{|c|}{ Criteria met } & \multicolumn{2}{|c|}{ Criteria not met } & \\
\hline & $\mathrm{n}$ & $\%$ & $\mathrm{n}$ & $\%$ & \\
\hline \multicolumn{6}{|c|}{ Traditional classification } \\
\hline Probable dengue fever & 213 & 89.5 & 25 & 10.5 & 238 \\
\hline Dengue fever confirmed & 134 & 62.9 & 79 & 37.1 & 213 \\
\hline Dengue hemorrhagic fever & 128 & 53.8 & 110 & 46.2 & 238 \\
\hline Dengue shock syndrome & 65 & 50.8 & 63 & 49.2 & 128 \\
\hline \multicolumn{6}{|c|}{ Revised classification } \\
\hline Dengue with/without warning signs & 210 & 99.1 & 2 & 0.9 & 212 \\
\hline Severe dengue & 113 & 53.3 & 99 & 46.7 & 212 \\
\hline
\end{tabular}


Table 2. Dengue fatality in terms of the 1997 and revised classification schemes.

\begin{tabular}{ccccc}
\hline & $\mathrm{n}$ & No. deaths & Rate & Fatality ratio $^{\mathrm{a}}$ \\
\hline Severe dengue declared & 238 & 16 & 6.7 & \\
Dengue hemorrhagic fever & 128 & 9 & 7.0 & 1.04 \\
Severe dengue & 113 & 13 & 11.5 & 1.72 \\
\hline
\end{tabular}

${ }^{a}$ Baseline category: severe dengue declared.

In this study, we sought to contribute to the analysis of different point estimators for fatality rates of dengue resulting from using different diagnostic criteria, by stressing the importance of clearly defining denominators [8]. The comparison of fatality rates between outbreaks at different times in endemic areas, or between different countries, could be invalid unless the definition criteria are subject to strict control. Obviously differences result from calculating fatality using all cases of possible dengue, compared to using only those considered cases on the basis of diagnostic criteria $[9,10]$. However, even using the revised classification, uncertainty about the strict application of its criteria may lead to variations in fatality rate as seen in this study (75\%). For the purposes of assessing fatality rate in an outbreak, this variation is excessive. A limitation of the study is that some of the definitions of signs and symptoms used to classify dengue as severe are ambiguous. Thus, the construction of some variables had to be based on others, for example, "severe bleeding as evaluated by clinician" was created based on a record of blood transfusion.

The assessment of fatality rate of dengue outbreaks, as a public health problem, ought to take stability in the definition of cases into account.

\section{ACKNOWLEDGEMENTS}

This study was supported by the Ecuadorian Public Health Ministry. PL and VR had grants from the Ministry of Health of Ecuador for the residency program of Family and Community Medicine, 2010-2013 period.

\section{DISCLAIMER}

The opinions expressed by authors contributing to this journal do not necessarily reflect the opinions of the Public Health Ministry of Ecuador or the institutions with which the authors are affiliated.

\section{REFERENCES}

[1] Torres, E.M. (2006) La prevención de la mortalidad por dengue: Un espacio y un reto para la atención primaria de salud. Revista Panamericana de Salud Pública, 20, 60-74. http://dx.doi.org/10.1590/S1020-49892006000700013

[2] World Health Organization (1997) Dengue haemorrhagic fever: Diagnosis, treatment, prevention and control. [Internet]. 2nd Edition, Geneva.

http://www.who.int/csr/resources/publications/dengue/De nguepublication/

[3] World Health Organization and Special Programme for Research and Training in Tropical Diseases (2012) Handbook for clinical management of dengue [Internet]. http://www.who.int/denguecontrol/9789241504713/en/in dex.html

[4] Horstick, O., Farrar, J., Lum, L., Martinez, E., San Martin, J.L., Ehrenberg, J., et al. (2012) Reviewing the development, evidence base, and application of the revised dengue case classification. Pathogens and Global Health, 106, 94-101. http://dx.doi.org/10.1179/2047773212Y.0000000017

[5] Kalayanarooj, S. (2011) Dengue classification: Current WHO vs. the newly suggested classification for better clinical application? Journal of the Medical Association of Thailand, 94, S74-S84.

[6] Boletín epidemiológico de la situacióndel dengue en el ecuador, No. 46, 07 de enero de 2013 [Internet]. Ministerio de Salud Publica.

http://www.salud.gob.ec/boletin-epidemiologico-de-la-situ acion-del-dengue-en-el-ecuador-no-46-07-de-enero-de-20 $\underline{13 /}$

[7] Aung, K.L.L., Thanachartwet, V., Desakorn, V., Chamnanchanunt, S., Sahassananda, D., Chierakul, W., et al. (2013) Factors associated with severe clinical manifestation of dengue among adults in Thailand. Southeast Asian Journal of Tropical Medicine and Public Health, 44, 602612.

[8] Narvaez, F., Gutierrez, G., Pérez, M.A., Elizondo, D., Nuñez, A., Balmaseda, A., et al. (2011) Evaluation of the traditional and revised who classifications of dengue disease severity. PLOS Neglected Tropical Diseases, 5, e1397. http://dx.doi.org/10.1371/journal.pntd.0001397

[9] Van de Weg, C.A.M., van Gorp, E.C.M., Supriatna, M., Soemantri, A., Osterhaus, A.D.M.E. and Martina, B.E.E. (2012) Evaluation of the 2009 WHO dengue case classification in an indonesian pediatric cohort. The American Journal of Tropical Medicine and Hygiene, 86, 166-170. http://dx.doi.org/10.4269/ajtmh.2012.11-0491

[10] Huy, N.T., Van Giang, T., Thuy, D.H.D., Kikuchi, M., Hien, T.T., Zamora, J., et al. (2013) Factors associated with dengue shock syndrome: A systematic review and meta-analysis. PLOS Neglected Tropical Diseases, 7, e2412. http://dx.doi.org/10.1371/journal.pntd.0002412 